\title{
Nucleotide excision repair deficiencies and the somatotropic axis in aging
}

\author{
George A. Garinis
}

Department of Genetics, Erasmus Medical Center, Rotterdam, the Netherlands

\begin{abstract}
The physicochemical constitution of DNA cannot warrant lifelong stability. Yet, unlike all other macromolecules, nuclear DNA must last the lifetime of a cell ensuring that its vital genetic information is preserved and faithfully transmitted to progeny. An increasing body of evidence suggests that progressive genome instability likely contributes to aging and shortens lifespan. In support, defects in genome surveillance pathways rapidly accelerate the onset of age-related pathology, including cancer. This review describes the role of DNA damage in aging along with a number of progeroid syndromes and associated mouse models with defects in nucleotide excision repair that age rapidly and die prematurely.
\end{abstract}

Key words: Aging, DNA damage, DNA repair, Progeria, Somatotropic axis

\section{THE CONSEQUENCES OF DNA DAMAGE}

DNA carries the genetic blueprint for all vital cellular instructions. Even so, nuclear DNA is not disposable and, unlike all other macromolecules, must last the lifetime of a cell. However, our genome is continuously exposed to the damaging effects of several harmful exogenous (chemical or physical) and/or endogenous agents. For instance, ultraviolet-B (UV-B) is a well-known universal carcinogen with an increasing impact due to depletion of the ozone layer as well as to natural and artificial sun tanning. ${ }^{1}$ As a consequence, skin cancer is steadily increasing. Exposure to UV light mainly introduces Cyclobutane Pyrimidine Dimers (CPDs) and (6-4)

Address for correspondence:

George A. Garinis, Dr Molewaterplein 50, 3015GE,

Rotterdam, the Netherlands, e-mail: g.garinis@erasmusmc.nl

Received 02-10-07, Revised 10-11-07, Accepted 05-12-07 photoproducts (6-4PPs) in the DNA generated by covalent bonds between two adjacent pyrimidines. ${ }^{2}$ Such lesions often interfere with biological processes critical for cell viability. For example, the presence of unrepairable UV-induced DNA lesions may hamper the process of transcription by blocking RNA synthesis, thus potentially preventing the generation of messenger RNA and encoded protein of a gene. As DNA lesions interfere with gene expression, they also affect a number of vital responses critical for the survival of a cell against hazardous threats. Furthermore, helix-distorting lesions also interfere with DNA replication. ${ }^{1}$ DNA damage-induced replication stress may often result in point mutations known to comprise a primary step in cancer initiation and a major cause of various inborn defects and overall cellular malfunctioning. Double-strand DNA breaks (DSBs) induced by X-rays, chemicals such as cisplatin that may also introduce cross-links or during replica- 
tion of Single-Strand Breaks (SSBs) are frequently responsible for numerous chromosomal aberrations, including aneuploidy, deletions and chromosomal translocations, all intimately linked to carcinogenesis (leukemia and lymphomas), particularly in those cell types (e.g. B- and T-cells) that are associated with specialized DNA recombination activities. ${ }^{3}$

Besides the numerous environmental and genotoxic agents that constantly damage our genome, it is the continuous threat posed by intrinsic sources of genome instability, such as inherent replication errors, spontaneous chemical disintegration (e.g. certain chemical bonds in DNA are intrinsically unstable due to spontaneous hydrolysis) and (by-)products of normal cellular metabolism, that offer an additional biologically relevant ground to genomic decay. The latter category includes the reactive oxygen species (ROS), including the superoxide anions, the hydrogen peroxide and hydroxyl radicals that are generated by mitochondria, peroxisomes and the cytochrome P450 as well as during the oxidative antimicrobial activity of phagocytic cells. ROS induce more than 100 different types of DNA injuries. ${ }^{4}$ Indeed, it has been estimated that in this way, about 10,000 lesions are induced in the genome of each cell per day. A notorious mutagenic injury is 8-oxodG, a major oxidative lesion that pairs equally well with the proper $\mathrm{C}$ as well as with the incorrect A during replication, causing high levels of GC to TA transversions.

Basically, different types of lesions have distinct consequences with respect to helix distortion as well as ability to suspend or obstruct DNA replication, block ongoing transcription or else hamper the battery of genome repair systems and caretakers that continuously safeguard the genome. Depending on the type of DNA damage, certain DNA injuries could trigger cell death via apoptosis or else induce (replicative) senescence (cytotoxic or cytostatic lesions, e.g. DSBs) or mutations (mutagenic lesions, e.g. CPDs). In the long term and depending on the type of damage, such lesions may result in point mutations and cancer or else fuel genome erosion with time, thereby interfering with the cellular metabolism and contributing to the process of age-related functional decline. The detrimental and adverse short- and long-term effects of DNA damage in overall organismal survival has forced the evolution of intricate defense mechanisms that continuously scan, detect and repair DNA injuries inflicted in our genome.

\section{DEFENSE MECHANISMS AND THE NUCLEOTIDE EXCISION REPAIR SYSTEM}

Before any attempt to restore the damaged DNA back to its original form takes place, cells employ a complex network of molecular and physical defense mechanisms that prevent or minimize the exposure of our genome to exogenous or endogenous sources of DNA damage. Not surprisingly, ROS as well as other metabolic products are thought to constitute a major and continuous endogenous source of damage that is not only limited to nucleic acids but additionally includes fatty acids, proteins and other biomolecules that gradually drive cellular degeneration and functional decline with time. This is why cells are invested with the capacity to substantially lessen the burden of oxidative metabolism by mounting an enormous antioxidant molecular defense system composed of enzymatic (superoxide dismutase, catalase, glutathione peroxidase and peroxyredoxins) as well as low molecular-mass scavengers (such as glutathione). Yet, free radicals are still capable of escaping this sophisticated defense barrier and damaging our genome. Moreover free radicals participate in processes that are also required for proper cellular function (e.g. cell signaling, the destruction of harmful bacteria by neutrophils) and benefit organismal fitness. ${ }^{5-8}$ Consequently, the damaging effects of ROS are unavoidable. Thus, cells rely on repair pathways that attempt to guarantee the life-long stability and proper function of DNA. Lesions affecting only one strand of the double helix are often the substrate of two major repair pathways: Base Excision Repair (BER) and Nucleotide Excision Repair (NER). Both pathways are mechanistically distinct and deal with fundamentally different types of lesions. Whereas BER deals with nucleotide alterations such as alkylated, deaminated and oxidized bases, NER is primarily involved in the repair of severely helix distorting injuries, such as bulky nucleotide adducts and intrastrand crosslinks that interfere with base pairing, thereby interfering with transcription and normal replication. ${ }^{3}$ This mode of repair operates via a "cut and patch" type of mechanism and proceeds in several consecutive steps: lesion recognition, local opening of the double 
helix and lesion verification, excision of the damage together with some flanking sequences and gap-filling DNA synthesis. ${ }^{9}$ Two modes of lesion recognition delineate distinct NER subpathways: global genome NER (GG-NER) that scans for DNA lesions on the entire genome and the transcription-coupled NER (TC-NER) that focuses on the transcribed strand of active genes. In GG-NER, the XPC-HR23B (the human rad23b homologue) complex possibly also enhanced by the DNA binding-E3-ubiquitin ligase, XPE (also called UV-DDB2), is responsible for the genome-wide detection of damage..$^{10-12}$ This step prompts the helicase subunits XPB, XPD and the core component $\mathrm{p} 8 / \mathrm{TTD}$-A of the repair/transcription complex TFIIH to open the double helix surrounding the lesion. ${ }^{13-15} \mathrm{XPA}$, a damage verification protein, coordinates and stabilizes the remaining NER proteins, whereas RPA binds and protects the single-stranded DNA regions formed during the "repair bubble". ${ }^{16}$ Next, the endonucleases ERCC1/XPF and XPG (3' and 5 ' exonucleases, respectively) excise a patch of 30 residues containing the damage. Subsequently, the regular replication machinery (DNA Pol $\varepsilon$ or $\delta$ ) resumes filling the gap, whereas the resulting nick is ligated, presumably by ligase I, thereby completing the reaction. However, in TC-NER the damage recognition step does not involve the XPC-HR23B complex. ${ }^{17,18}$ Instead, it has been proposed that TCNER is likely initiated by stalling of an elongating RNA polymerase at a lesion during ongoing transcription, in addition to the TC-NER-associated factors CSA and CSB proteins. CSA can be found in a complex containing the cullin-based ubiquitin E3 ligase, which is regulated by the COP9 signalosome complex of the ubiquitin-proteasome pathway ${ }^{19}$ as well as in other protein complexes containing RNA pol II (e.g. TFIIH or XAB2, a tetratricopeptide repeat protein involved in TC-NER and transcription). ${ }^{20,21}$ Even though CSA does not co-localize with CSB in vivo, they both interact in vitro. ${ }^{20} \mathrm{CSB}$ (also known as Ercc6) is a DNA-dependent ATPase and chromatin remodelling factor belonging to the SWI/SNF2 family of DNA-dependent ATPases. ${ }^{22,23}$ Although its exact role in TC-NER remains unknown, it has been suggested that CSB might interact with the transcriptional machinery by transiently binding elongating RNA pol II, thereby verifying that the transcription proceeds normally. ${ }^{16,24}$ Once released, the RNA pol II can either be degraded by the CSA complex or else be recycled after dephosphorylation by FCP1, the subunit of human protein phosphatase that dephosphorylates the carboxy-terminal domain of RNA polymerase IIO..$^{25}$ TCR, part of which is TC-NER, appears not restricted to NER and can remove transcription-blocking lesions from the transcribed strand of active genes. ${ }^{26,27}$ For instance, limited evidence suggests that CSB protein might not only be restricted to TC-NER but also acts in other repair pathways (e.g. BER) aimed at repairing (non-NER) lesions that could still interfere with ongoing transcription. ${ }^{28-30}$ However, despite the importance of these early experiments, the findings reported therein describe protein interactions observed either in in vitro experimental conditions or else in cultured cell systems (e.g. HeLa cells), but not in the intact organism. In addition, it has previously been suggested that TCR might also apply to oxidative DNA lesions, normally only removed by BER, a likely scenario that has not, however, so far been formally proven. ${ }^{31-33}$ Interestingly, the activity of NER may actually vary with respect to the cell type involved. For instance, GG-NER can be attenuated relatively early during differentiation. ${ }^{34}$ This has led to the speculation that terminally differentiated cells (e.g. neurons) do not need to replicate their genome and, therefore, could dispense with the task of removing DNA damage on a global genome scale. Be that as it may, neurons would still need to maintain the genomic integrity of genes that are actively being transcribed $^{34,35}$ by employing TCR that relies on the template (non-transcribed) strand.

\section{NER PROGEROID SYNDROMES}

Over the last decade, there has been substantial evidence that accumulation of (nuclear) DNA damage contributes to the onset of age-related pathology, including cancer. In humans, a number of progeroid disorders with inherited defects in NER or in other, distinct from NER, genome surveillance pathways support the causal role of genome instability in aging. ${ }^{36-38}$ For example, Cockayne Syndrome (CS; affected proteins: CSB, CSA), Trichothiodystrophy (TTD; affected proteins: XPB, XPD, TTDA) or XPF-ERCC1 syndrome (XFE; affected proteins: XPF, ERCC1) represent progeroid conditions caused by defects in the highly conserved NER. ${ }^{15,41,43,47} \mathrm{CS}$ is a 
photosensitive disorder, originating from a defect in the Transcription-Coupled Repair (TCR) subpathway of NER that removes transcription-blocking lesions from the transcribed strand of active genes ${ }^{27}$ and likely also applies to transcription-blocking oxidative DNA lesions, ${ }^{32,39}$ which are removed primarily by Base Excision Repair. CS is accompanied by cachectic dwarfism (growth failure), delayed psychomotor development, mental retardation, microcephaly, ataxia, sensorineural hearing loss, retinal degeneration (categorized as progressive neurological abnormalities), along with impaired sexual development, kyphosis, osteoporosis and severely reduced lifespan (mean lifespan: 12.5 years). ${ }^{40,41}$ TTD patients are partially defective in TCR, as well as in the global genome repair subpathway of NER, and share several symptoms associated with CS. In addition, due to a partial defect in transcription itself, these patients also present with additional symptoms such as ichthyosis and brittle hair and nails. ${ }^{42}$ Many of the CS and TTD features appear gradually with age and resemble progerias. Moreover, the first case of human inherited ERCC1 deficiency was recently reported. ${ }^{43}$ Cells derived from this Ercc1-XPF patient showed moderate hypersensitivity to ultraviolet rays and mitomycin $\mathrm{C}$, yet the clinical features were severe and also compatible with a diagnosis of cerebro-oculo-facio-skeletal syndrome. Together, CS, TTD as well as Ercc1-XPF are often considered "segmental progeroid syndromes", since patients with these syndromes develop certain but not all aspects of natural aging early in life. ${ }^{37}$

\section{NER PROGEROID MOUSE MODELS}

Mouse models carrying defects in CS-A and CS-B genes consistently mimic the sensitivity of CS patients to UV and demonstrate accelerated photoreceptor loss, reduced body weight and mild neurodegeneration. ${ }^{44,45}$ Likewise, mice carrying a homozygous point mutation in the Xpd gene exhibit very similar features with those seen in TTD patients, ${ }^{46}$ including brittle hair, osteoporosis, osteosclerosis, kyphosis, cachexia and a reduced lifespan. ${ }^{36}$ Importantly, complete NER inactivation (by concurrent inactivation of the Xpa gene) considerably exacerbates the CS features of partially NER-defective TTD mice. For instance, XpdTTD/TTD/Xpa-/- double mutant animals display dramatic postnatal growth attenuation, kypho- sis, ataxia, abnormal locomotor activity as well as progressive weight loss and die prematurely before weaning. ${ }^{36}$ Likewise, $E r c c 1^{-/}$mice (carrying a defect in an endonuclease required for NER as well as for repair of the very cytotoxic DNA interstrand crosslinks) strikingly mimic a new syndrome, designated XFE (XPF-ERCC1) progeria. ${ }^{47}$ These animals demonstrate most of the previously describe progeroid features but also others that are, in part, distinct from mice deficient only in TCR such as dramatic liver, kidney and bone marrow pathology. This suggests that defects in different repair systems for cytotoxic lesions might trigger a different spectrum of progeroid symptoms. The notion that different types of lesions and associated repair systems are implicated in distinct age-related pathologies could also explain the segmental nature of NER progerias. The early onset of progeroid features in DNA repair-deficient mice along with the ability of DNA lesions to provoke permanent cell cycle arrest or apoptosis led to the hypothesis that aspects of what we perceive as "age-related deterioration" might, in fact, originate from (oxidative) DNA lesions that eventually obstruct basic biological processes such as transcription and/or replication, thereby leading to (programmed) cell death or senescence and ultimately to loss of tissue homeostasis and onset of age-related deterioration. ${ }^{48-50}$

\section{DNA DAMAGE AND THE GROWTH HORMONE (GH)/INSULIN GROWTH FACTOR 1 (IGF1) AXIS}

Unpredictably, however, recent findings in a number of short-lived, DNA repair-deficient animals that show a series of remarkable progeroid features revealed numerous metabolic and growth changes associated with delayed aging and extended lifespan as seen in dwarf mutants and calorie restricted mice. ${ }^{36,47,51}$ Mouse liver transcriptome analysis and/or various physiological endpoints in a series of NER-deficient mouse models with demonstrated progeria $\left(\mathrm{Csb}^{\mathrm{m} / m} / \mathrm{Xpa}^{-/}, \operatorname{Ercc~}^{-/}\right.$and $\mathrm{Xpd}^{\mathrm{TTD}} / \mathrm{Xpa}^{-/-}$mouse mutants) demonstrated: i) a profound attenuation of the somatotroph axis as evidenced by the consistent suppression of genes encoding central components of the GH/IGF1 axis; ii) decreased expression of several genes associated with mitogenic signals as well as catabolism such as key components of the glycolysis, tricarboxylic acid cycle and additional oxidative phosphorylation pathways 
(i.e. several cytochrome $\mathrm{P} 450$ monooxygenases, the $\mathrm{NADH}$ dehydrogenase complex and the NADPHdependent oxidative metabolism); iii) a significant up-regulation of genes associated with glycogen synthesis, i.e. Gyg1 and Gys 2 and down-regulation of glycogen phosphorylase $(P y g l)$, involved in the breakdown of glycogen into its constitutive glucose monomers; and iv) the substantial down-regulation in the expression of genes coupled with peroxisomal metabolism and biosynthesis. Clearly, the complete catabolic metabolism appeared to be restrained in the liver of NER progeroid mice. Furthermore, these findings revealed that NER progeroid mice store rather than burn glucose monomers for derivation of energy, an observation that at first appeared contradictory to their early age at a time when wild type animals attempt to maximize the utilization of energy reserves for growth and development.

The above expression changes were coupled with the broad up-regulation in gene expression of genes associated with fatty acid synthesis and transport along with the up-regulation of the receptor for the adipocyte hormone leptin ( $L p l r)$ and the central fat regulator peroxisome proliferator-activated receptor-gamma (Ppary). Surprisingly, similar to the previously documented limited glucose utilization and enhanced glycogen synthesis, NER progeroid mice demonstrated a propensity to store rather than burn fat, an additional major reservoir. Further detailed analysis in the transcriptomes of NER-deficient animals with accelerated aging revealed an up-regulation of genes encoding key enzymatic and non-enzymatic low molecular mass scavengers and antioxidant defense enzymes along with components of the glutathione system, likely revealing the effort of NER progeroid mice to minimize the induction of (DNA) damage by counteracting ROS.

Importantly, several of these findings in the transcriptomes of NER progeroid mutants were further confirmed by serum measurements and immunostaining of liver tissue sections demonstrating substantially lower IGF1, insulin and glucose serum levels, a lower enzymatic activity of citrate synthase in the liver as well as enhanced accumulation of glycogen in unusually large vesicles and of triacylglycerides in the 2-week old NER progeroid mouse livers as compared to wild type littermate controls. Also significantly, the increased glycogen accumulation did not derive from an inability to split glycogen into its glucose monomers. Overnight fasting in the $C s b^{m / m} / X_{p a}^{-/}$pups and littermate controls (by taking away the lactating mother as well as food pellets) resulted in a near-to-complete depletion of liver glycogen.

How similar were the expression changes of NER progeroid mice to those observed in naturally aged animals? Comparative gene expression analysis between the livers of 2-week old $\mathrm{Csb}^{m / m} / \mathrm{Xpa}^{-/-}$and $\mathrm{Ercc}^{-/-}$mice and those derived from naturally aged 130 -week old wild type mice revealed broad genome-wide parallels between the transcriptomes of NER progeroid and naturally aged liver tissues, thereby strongly supporting the notion that these changes are intrinsic to natural aging as well as NER progeria. Therefore, $\mathrm{Csb}^{m / m} / \mathrm{Xpa}^{-/-}$and $E r c c 1^{-/-}$mice recapitulate the shift in the hormonal, oxidative metabolism and anti-oxidant defense response, closely mimicking the natural process of aging.

Paradoxically, however, the suppression of the somatotroph, lactotroph and thyrotroph axes along with the concomitant suppression of oxidative metabolism and the up-regulation of antioxidant responses evidenced in short-lived NER progeroid mice are also manifested by long-lived Snell and Ames dwarf and calorie-restricted animal models. ${ }^{52,53}$ This pointed to the hypothesis that the inherent DNA repair defect, and thus the gradual but rapid accumulation of DNA damage in the genome of these mice, triggers a series of highly conserved, physiological homeostatic responses that likely favour longevity, a notion that also conforms to the nematode longevity paradigm,,$^{53}$ the long-lived dwarf mutant and calorie restricted mice. ${ }^{54-56}$ This also implies that a response previously associated with prolonged lifespan can be induced upon genotoxic stress, even early in life. At present, the most likely scenario on how repair-deficient mice would benefit from such a response lies in the potential link between the harmful effects of metabolism and that of progressive genome instability. During development, the mitogenic action of GH and IGF1 likely promotes the activity of (oxidative) metabolism that drives organismal growth. ${ }^{55,57,58}$ If so, however, an intense metabolic activity is thought to lead to higher oxygen consumption ${ }^{55}$ and, as a result, would also increase the generation of free radicals through 
the concerted increased activity of mitochondrial electron transport, peroxisomal fatty acid metabolism and/or microsomal cytochrome $P-450$ enzymes. ${ }^{59}$ Cells attempt to lessen the harmful effects of ROS by mounting a battery of anti-oxidant, defense and DNA repair mechanisms. However, oxidative DNA damage is still expected to accumulate, leading to the impairment of the previously discussed biological processes (i.e. transcription, replication) and leading to cellular senescence, malfunction or death and eventually to progressive loss of tissue homeostasis and organismal decline. Hence, the findings support the notion that complete NER abrogation (by inactivation of $X p a$ ) renders TCR-deficient $C s b^{m / m}$ mice and $\mathrm{ErcC}^{-/}$mice (that are also deficient in the repair of cross-links) capable of coping with the increased burden of DNA damage, resulting in the initiation of an adaptive response (reduction of metabolic activity through down-regulation of the GH/IGF1 axis) to relieve the pressure on their genome. This response, therefore, appears to be a futile attempt to extend the limited lifespan of the NER progeroid mice, revealing a link between damage to the genome and regulation of the GH/IGF1 hormonal axis. As a consequence, however, the initially physiological pace of growth diminishes soon after birth (as levels of key hormones associated with growth decline), eventually leading to severe growth retardation and ultimately to dramatically accelerated progeria. Alternatively, in view of the damage accumulated in their genome, a continuous activity of mitogenic signals is liable to greatly increase the chance of tumour initiation and dramatic tissue pathology rapidly after birth.

If the presence of unrepairable DNA damage is capable of inducing the enormity of observed metabolic changes, there was speculation as to whether similar types of responses (e.g. the GH/IGF1 suppression) to those seen in DNA repair-deficiency (that rapidly accumulate unrepaired DNA damage) and naturally aged mice (where damage accumulates gradually over time) could also be seen in wild type animals exposed to genotoxic stress. If so, this would provide substantial proof of the role of DNA damage in physiological changes also observed in natural aging. Indeed, exposure of wild type mice chronically to low doses of genotoxic agents (e.g. DEHP or mitomycin that induces oxidative stress or cross-links, respectively) triggered a similar suppression of the somatotroph, thyrotroph and lactotroph axes along with the up-regulation of antioxidant responses, data pointing to a link between genome instability and the age-related decline of the $\mathrm{GH} / \mathrm{IGF} 1$ somatotroph axis.

Thus, accumulation of persisting, endogenous DNA damage comprises a root cause of the decline of the GH/IGF1 hormonal axis and the consequent organismal deterioration with advancing aging. Resultantly, prematurely aging mice, deficient in nucleotide excision and transcription-coupled repair and/or cross link repair, provide strong evidence for the role of DNA damage in promoting aging, disclosing a novel link between inherent genome instability, the physiologic decline of GH/IGF1 somatotroph axis and the organismal deterioration with advancing age. Future research will likely focus on the molecular mechanism that connects DNA damage with the GH/IGF1 hormonal response and on modes of intervention in the dramatic pace of aging in the repair-compromised mouse mutants.

\section{ACKNOWLEDGMENTS}

I wish to thank Dr. Gijsbertus van der Horst, Dr. Bjorn Schumacher, Dr. Laura Niedernhofer and Dr. Jan Hoeijmakers for critically discussing the ideas and concepts presented in this manuscript. This work was supported by the Netherlands Organization for Scientific Research (NWO) through the foundation of the Research Institute Diseases of the Elderly, as well as grants from SenterNovem IOP-Genomics (IGE03009), NIH (1PO1 AG17242-02), NIEHS (1UO1 ES011044), EC (QRTL-1999-02002) and the Dutch Cancer Society (EUR 99-2004).

\section{REFERENCES}

1. Friedberg EC, Walker GC, Siede W, 1995 DNA Repair and Mutagenesis. San Francisco, WH Freeman and Company.

2. Mitchell DL, 1988 The relative cytotoxicity of (6-4) photoproducts and cyclobutane dimers in mammalian cells. Photochem Photobiol 48: 51-57.

3. Hoeijmakers JH, 2001 Genome maintenance mechanisms for preventing cancer. Nature 411: 366-374.

4. Cadet J, Berger M, Douki T, Ravanat JL, 1997 Oxidative damage to DNA: formation, measurement, and biological significance. Rev Physiol Biochem Pharmacol 131: 1-87. 
5. Finkel T, 2001 Reactive oxygen species and signal transduction. IUBMB Life 52: 3-6.

6. Klein C, 2002 Nitric oxide and the other cyclic nucleotide. Cell Signal 14: 493-498.

7. Masters CJ, 1996 Cellular signalling: the role of the peroxisome. Cell Signal 8: 197-208.

8. Hampton MB, Kettle AJ, Winterbourn CC, 1998 Inside the neutrophil phagosome: oxidants, myeloperoxidase, and bacterial killing. Blood 92: 3007-3017.

9. Hoeijmakers JH, 2001 DNA repair mechanisms. Maturitas 38: $17-22$.

10. Tang JY, Hwang BJ, Ford JM, Hanawalt PC, Chu G, 2000 Xeroderma pigmentosum p48 gene enhances global genomic repair and suppresses UV-induced mutagenesis. Mol Cell 5: 737-744.

11. Tang J, Chu G, 2002 Xeroderma pigmentosum complementation group E and UV-damaged DNA-binding protein. DNA Repair (Amst) 1: 601-616.

12. Sugasawa K, Ng JM, Masutani C, et al, 1998 Xeroderma pigmentosum group $\mathrm{C}$ protein complex is the initiator of global genome nucleotide excision repair. Mol Cell 2: 223-232.

13. Riedl T, Hanaoka F, Egly JM, 2003 The comings and goings of nucleotide excision repair factors on damaged DNA. Embo J 22: 5293-5303.

14. Coin F, Proietti De Santis L, Nardo T, Zlobinskaya O, Stefanini M, Egly JM, 2006 p8/TTD-A as a repair-specific TFIIH subunit. Mol Cell 21: 215-226.

15. Giglia-Mari G, Coin F, Ranish JA, 2004 A new, tenth subunit of TFIIH is responsible for the DNA repair syndrome trichothiodystrophy group A. Nat Genet 36: 714-719.

16. Laine JP, Egly JM, 2006 When transcription and repair meet: a complex system. Trends Genet 22: 430-436.

17. Mellon I, Bohr VA, Smith CA, Hanawalt PC, 1986 Preferential DNA repair of an active gene in human cells. Proc Natl Acad Sci U S A 83: 8878-8882.

18. Bohr VA, Smith CA, Okumoto DS, Hanawalt PC, 1985 DNA repair in an active gene: removal of pyrimidine dimers from the DHFR gene of CHO cells is much more efficient than in the genome overall. Cell 40: 359-369.

19. Schwechheimer C, 2004 The COP9 signalosome (CSN): an evolutionary conserved proteolysis regulator in eukaryotic development. Biochim Biophys Acta 1695: 45-54.

20. Henning KA, Li L, Iyer N, et al, 1995 The Cockayne syndrome group A gene encodes a WD repeat protein that interacts with CSB protein and a subunit of RNA polymerase II TFIIH. Cell 82: 555-564.

21. Nakatsu Y, Asahina H, Citterio E, et al, 2000 XAB2, a novel tetratricopeptide repeat protein involved in transcription-coupled DNA repair and transcription. J Biol Chem 275: 34931-34937.

22. Citterio E, Van Den Boom V, Schnitzler G, et al, 2000 ATP-dependent chromatin remodeling by the Cockayne syndrome B DNA repair-transcription-coupling factor. Mol Cell Biol 20: 7643-7653.
23. Henning KA, Li L, Iyer N, et al, 1995 The Cockayne syndrome group A gene encodes a WD repeat protein that interacts with CSB protein and a subunit of RNA polymerase II TFIIH. Cell 82: 555-564.

24. van den Boom V, Citterio E, Hoogstraten D, et al, 2004 DNA damage stabilizes interaction of CSB with the transcription elongation machinery. J Cell Biol 166: 27-36.

25. Archambault J, Pan G, Dahmus GK, et al, 1998 FCP1, the RAP74-interacting subunit of a human protein phosphatase that dephosphorylates the carboxyl-terminal domain of RNA polymerase IIO. J Biol Chem 273: 27593-27601.

26. Hanawalt PC, Donahue BA, Sweder KS, 1994 Repair and transcription. Collision or collusion? Curr Biol 4: 518-521.

27. Hanawalt PC, 2002 Subpathways of nucleotide excision repair and their regulation. Oncogene 21: 8949-8956.

28. Tuo J, Chen C, Zeng X, Christiansen M, Bohr VA, 2002 Functional crosstalk between hOgg1 and the helicase domain of Cockayne syndrome group B protein. DNA Repair (Amst) 1: 913-927.

29. Thorslund T, von Kobbe C, Harrigan JA, et al, 2005 Cooperation of the Cockayne syndrome group $\mathrm{B}$ protein and poly(ADP-ribose) polymerase 1 in the response to oxidative stress. Mol Cell Biol 25: 7625-7636.

30. Stevnsner T, Nyaga S, de Souza-Pinto NC, et al, 2002 Mitochondrial repair of 8-oxoguanine is deficient in Cockayne syndrome group B. Oncogene 21: 8675-8682.

31. Dianov G, Bischoff C, Sunesen M, Bohr VA, 1999 Repair of 8-oxoguanine in DNA is deficient in Cockayne syndrome group B cells. Nucleic Acids Res 27: 1365-1368.

32. de Waard H, de Wit J, Gorgels TG, et al, 2003 Cell typespecific hypersensitivity to oxidative damage in CSB and XPA mice. DNA Repair (Amst) 2: 13-25.

33. Spivak G, Hanawalt PC, 2006 Host cell reactivation of plasmids containing oxidative DNA lesions is defective in Cockayne syndrome but normal in UV-sensitive syndrome fibroblasts. DNA Repair (Amst) 5: 13-22.

34. Nouspikel T, Hanawalt PC, 2002 DNA repair in terminally differentiated cells. DNA Repair (Amst) 1: 59-75.

35. Nouspikel T, Hanawalt PC, 2003 When parsimony backfires: neglecting DNA repair may doom neurons in Alzheimer's disease. Bioessays 25:168-173.

36. de Boer J, Andressoo JO, de Wit J, et al, 2002 Premature aging in mice deficient in DNA repair and transcription. Science 296: 1276-1279.

37. Martin GM, 2005 Genetic modulation of senescent phenotypes in Homo sapiens. Cell 120: 523-532.

38. Martin GM, Oshima J, 2000 Lessons from human progeroid syndromes. Nature 408: 263-266.

39. Spivak G, Hanawalt PC, 2006 Host cell reactivation of plasmids containing oxidative DNA lesions is defective in Cockayne syndrome but normal in UV-sensitive syndrome fibroblasts. DNA Repair (Amst) 5: 13-22.

40. Nance MA, Berry SA, 1992 Cockayne syndrome: review of 140 cases. Am J Med Genet 42: 68-84. 
41. Bootsma D, Kraemer KH, J.E. C, Hoeijmakers J, 2002 Nucleotide excision repair syndromes: Xeroderma Pigmentosum, Cockayne Syndrome and trichthiodystrophy. New York, McGraw-Hill Medical Publishing Division, pp, 211-237.

42. Vermeulen W, Rademakers S, Jaspers NG, et al, 2001 A temperature-sensitive disorder in basal transcription and DNA repair in humans. Nat Genet 27: 299-303.

43. Jaspers NG, Raams A, Silengo MC, et al, 2007 First reported patient with human ERCC1 deficiency has cerebro-oculo-facio-skeletal syndrome with a mild defect in nucleotide excision repair and severe developmental failure. Am J Hum Genet 80: 457-466.

44. van der Horst GT, van Steeg H, Berg RJ,et al, 1997 Defective transcription-coupled repair in Cockayne syndrome $\mathrm{B}$ mice is associated with skin cancer predisposition. Cell 89: 425-435.

45. van der Horst GT, Meira L, Gorgels TG,et al, 2002 UVB radiation-induced cancer predisposition in Cockayne syndrome group A (Csa) mutant mice. DNA Repair (Amst)1: 143-157.

46. de Boer J, Donker I, de Wit J, Hoeijmakers JH, Weeda G, 1998 Disruption of the mouse xeroderma pigmentosum group D DNA repair/basal transcription gene results in preimplantation lethality. Cancer Res 58: 89-94.

47. Niedernhofer LJ, Garinis GA, Raams A, et al, 2006 A new progeroid syndrome reveals that genotoxic stress suppresses the somatotroph axis. Nature 444: 1038-1043.

48. Hasty P, Campisi J, Hoeijmakers J, van Steeg H, Vijg J, 2003 Aging and genome maintenance: lessons from the mouse? Science 299: 1355-1359.

49. de Boer J, Hoeijmakers JH, 2000 Nucleotide excision repair and human syndromes. Carcinogenesis 21: 453460.

50. Mitchell JR, Hoeijmakers JH, Niedernhofer LJ, 2003 Divide and conquer: nucleotide excision repair battles cancer and ageing. Curr Opin Cell Biol 15: 232-240.

51. van der Pluijm I GG, Brandt RMC, Gorgels TGMF, et al, 2006 Impaired Genome Maintenance Suppresses the Growth Hormone-Insulin-Like Growth Factor 1 Axis in Mice with Cockayne Syndrome. PLoS Biol 5: 23-38.

52. Carter CS, Ramsey MM, Ingram RL, et al, 2002 Models of growth hormone and IGF-1 deficiency: applications to studies of aging processes and life-span determination. J Gerontol A Biol Sci Med Sci 57: B177-188.

53. Longo VD, Finch CE, 2003 Evolutionary medicine: from dwarf model systems to healthy centenarians? Science 299: 1342-1346.

54. Bartke A, Brown-Borg H, 2004 Life extension in the dwarf mouse. Curr Top Dev Biol 63: 189-225.

55. Carter CS, Ramsey MM, Sonntag WE, 2003 A critical analysis of the role of growth hormone and IGF-1 in aging and lifespan. Trends Genet 2002, 18:295-301

56. Brown-Borg HM, 2003 Hormonal regulation of aging and life span. Trends Endocrinol Metab 14: 151-153.

57. Bartke A, 2003 Is growth hormone deficiency a beneficial adaptation to aging? Evidence from experimental animals. Trends Endocrinol Metab 14: 340-344.

58. Chandrashekar V, Zaczek D, Bartke A, 2004 The consequences of altered somatotropic system on reproduction. Biol Reprod 71: 17-27.

59. Beckman KB, Ames BN, 1998 The free radical theory of aging matures. Physiol Rev 78: 547-581. 